\title{
Weights of partitions and character zeros
}

\author{
Christine Bessenrodt \\ Fachbereich Mathematik, Universität Hannover \\ Welfengarten 1, D-30167 Hannover, Germany \\ bessen@math. uni-hannover.de \\ Jørn B. Olsson \\ Matematisk Institut, Københavns Universitet \\ Universitetsparken 5,DK-2100 Copenhagen Ø, Denmark \\ olsson@math.ku.dk
}

Submitted: Jun 26, 2004; Accepted: Sep 13, 2004; Published: Sep 20, 2004

Mathematics Subject Classifications: 20C30, 05A17

Dedicated to our friend Richard Stanley

\begin{abstract}
We classify partitions which are of maximal $p$-weight for all odd primes $p$. As a consequence, we show that any non-linear irreducible character of the symmetric and alternating groups vanishes on some element of prime order.
\end{abstract}

\section{Introduction}

A well-known result by Burnside states that any non-linear irreducible character of a finite group vanishes on some element of the group. Recently this was refined in [5], where it was shown that such a character always has a zero at an element of prime power order. Moreover, it had been noticed in [5] that any non-linear irreducible character of a finite simple group except possibly the alternating groups even vanishes on some element of prime order. Here we show that this character property also holds for the alternating and the symmetric groups. Indeed, this vanishing property is a simple consequence of a combinatorial result on the weights of partitions, which may be of independent interest. The elements of prime order $p$ which we are going to use in the symmetric group $S_{n}$ are those of maximal weight, i.e., they are a product of $\left\lfloor\frac{n}{p} \mid p\right.$-cycles. (Here $\lfloor\cdot\rfloor$ denotes the floor function. Thus $\lfloor x\rfloor$ is the integral part of $x \in \mathbb{R}$.)

Consider a partition $\lambda=\left(\lambda_{1}, \lambda_{2}, \ldots, \lambda_{m}\right)$ of the integer $n$. For a given integer $r \in \mathbb{N}$, we denote by $w_{r}(\lambda)$ the $r$-weight of $\lambda$, i.e., $w_{r}(\lambda)$ is the maximal number of $r$-hooks that can successively be removed from $\lambda$. The resulting partition after removing this maximal 
number of $r$-hooks is then the $r$-core $\lambda_{(r)}$ of $\lambda$ (see [4] or [6] for details). In this paper, we will only deal with the case where $r$ is a prime number $p$. We present a classification of the partitions which have maximal $p$-weight $\left\lfloor\frac{n}{p}\right\rfloor$ for all (odd) primes $p$.

The relevance of this classification to the question about the vanishing of character values is easily explained: The irreducible characters of $S_{n}$ are labelled by the partitions $\lambda$ of $n$. The Murnaghan-Nakayama formula for character values in $S_{n}$ shows that the irreducible character labelled by $\lambda$ vanishes on a $p$-element of maximal weight, if the $p$-weight of $\lambda$ is not maximal.

Our main result is then the following:

Theorem 1.1 Let $\lambda$ be a partition of $n \in \mathbb{N}$. Then the following holds:

(1) $\lambda$ is of maximal p-weight for all primes $p$, if and only if one of the following occurs:

$$
\lambda=(n),\left(1^{n}\right) \text { or }\left(2^{2}\right) .
$$

(2) $\lambda$ is of maximal $p$-weight for all primes $p>2$, if and only if $\lambda$ is one of the partitions in $(1)$, one of $(n-1,1),\left(2,1^{n-2}\right)$, where $n=2^{a}+1$ for some $a \in \mathbb{N}$, or one of the following occurs:

$$
\begin{array}{ll}
n=6: & \lambda=(3,2,1) \\
n=8: & \lambda=(5,2,1) \text { or }\left(3,2,1^{3}\right) \\
n=9: & \lambda=(6,3) \text { or }\left(2^{3}, 1^{3}\right) \\
n=10: & \lambda=(4,3,2,1)
\end{array}
$$

This combinatorial classification result has the following consequence:

Theorem 1.2 Let $n \in \mathbb{N}$. Let $\chi$ be any non-linear irreducible character of the symmetric group $S_{n}$ or the alternating group $A_{n}$. Then $\chi$ vanishes on some element of prime order. If $\chi(1)$ is not a 2-power, then $\chi$ is zero on some element of odd prime order.

Remark 1.3 If an irreducible character $\chi$ of a finite group $G$ has a zero at an element of prime order $p$, then $p$ divides $\chi(1)$; thus we can only expect zeros of odd prime order if $\chi(1)$ is not a 2-power. Note that the irreducible characters of $S_{n}$ and $A_{n}$ of prime power degree have been classified in [1]; from Theorem 1.1 we can immediately recover the classification of irreducible characters of 2-power degree for these groups.

The converse of the statement above does not hold, even for $G=S_{n}$. We denote by $[\lambda]$ the irreducible character of $S_{n}$ associated to the partition $\lambda$. Then $[5,2](1)=14$, but $[5,2]$ does not vanish on any element of order 2 . This is not just an accident for the prime 2 : $\left[8,1^{3}\right](1)=120$, but $\left[8,1^{3}\right]$ has no zero on elements of order 2 or 3 .

Theorem 1.2 then allows us to settle the question left open in [5]. Combined with corresponding results on sporadic groups [5, Theorem 3.4] and simple groups of Lie type [5, Theorem 5.1] we obtain:

Corollary 1.4 Any non-trivial irreducible character of a finite simple group vanishes on some element of prime order. 
We refer to [4, section 2.5] for the labelling of the irreducible characters of $A_{n}$. Now [4, 6.2.45] gives a simple relation between the $p$-weight of a partition $\lambda$ and the defect of the $p$-block containing the irreducible character labelled by $\lambda$. Therefore the following is another consequence of Theorem 1.1:

Theorem 1.5 (1) The characters $[n],\left[1^{n}\right]$ and $\left[2^{2}\right]$ are the only irreducible characters of $S_{n}$ which are in $p$-blocks of maximal defect for all primes $p$.

Apart from $\left[1^{2}\right],\left[1^{3}\right],\left[1^{4}\right],\left[1^{6}\right],\left[2^{2}\right]$, the trivial character of $S_{n}$ is the only irreducible character which is in the principal $p$-block for all primes $p \leq n$.

(2) The characters $\{n\},\{2,1\}_{ \pm}$and $\left\{2^{2}\right\}_{ \pm}$are the only irreducible characters of $A_{n}$ which are in p-blocks of maximal defect for all primes $p$.

They belong to the principal $p$-block for all primes $p \leq n$, except for the characters $\{2,1\}_{ \pm}$ at $p=2$.

The paper is organized as follows. Section 2 contains some results on hook lengths and on large prime divisors of products of consecutive integers. In section 3 we consider partitions of maximal weight and modify an algorithm of [1] to suit our purposes better. The algorithms are used to generate "large" first column hook lengths in the partitions under consideration. The final section contains the proofs of all the results stated above.

\section{Preliminaries}

We refer to [4], [6] for details about partitions, Young diagrams and hooks. Consider a partition $\lambda=\left(\lambda_{1}, \lambda_{2}, \ldots, \lambda_{l}\right)$ of the integer $n$. Thus $\lambda_{1} \geq \lambda_{2} \geq \ldots \geq \lambda_{l}>0$ and $\lambda_{1}+\lambda_{2}+\ldots+\lambda_{l}=n$. We call the $\lambda_{i}$ 's the parts of $\lambda$ and $l=l(\lambda)$ the length of $\lambda$. Moreover for $i \geq 1, m_{i}=m_{i}(\lambda)$ denotes the number of parts equal to $i$ in $\lambda$. The Young diagram of $\lambda$ consists of $n$ nodes (boxes) with $\lambda_{i}$ nodes in the $i$ th row. We refer to the nodes in matrix notation, i.e. the $(i, j)$-node is the $j$ th node in the $i$ th row. The $(i, j)$-hook consists of the nodes in the Young diagram to the right of and below the $(i, j)$-node, and including this node. The number of nodes in this hook is its hook length, denoted by $h_{i j}$. For the hook lengths in the first column we write $h_{i}=h_{i 1}, i=1, \ldots, l$; these first column hook lengths of $\lambda$ will play an important rôle in our investigation.

We will often make use of the following property of the weight of a partition (see [4, 2.7.37], [6, (3.3)]).

Lemma 2.1 If $\lambda$ is a partition of p-weight $w_{p}(\lambda)=w$, then $\lambda$ has exactly $w$ hooks of length divisible by $p$. In particular, if $\lambda$ has a hook of length divisible by $p$, then it has a hook of length $p$.

We also recall some elementary results about hook lengths from [1, section 2].

Proposition 2.2 Let $\lambda$ be a partition of $n, l=l(\lambda)$. Let $1 \leq i<j \leq l$. Then

$$
h_{i}+h_{j} \leq n+1+m_{1} .
$$


Lemma 2.3 Let $\lambda$ be a partition of $n$. Suppose that $s=h_{i k}$ and $t=h_{j m}$ where $(i, k) \neq$ $(j, m)$.

(1) If $i \neq j$ and $k \neq m$, then $s+t \leq n$.

(2) If $s+t>n$, then either $i=j=1$ (both hooks in the first row) or $k=m=1$ (both hooks in the first column).

Corollary 2.4 Let $\lambda$ be a partition of $n$. For $j \geq 2$, every hook length $t>n-h_{j}=n-h_{j 1}$ is a first column hook length of $\lambda$.

We will also need some number-theoretic results. Improving on a result by Sylvester and Schur, Hanson [2] proved the following:

Theorem 2.5 The product of $k$ consecutive numbers all greater than $k$ contains a prime divisor greater than $\frac{3}{2} k$, with the only exceptions $3 \cdot 4,8 \cdot 9$ and $6 \cdot 7 \cdot 8 \cdot 9 \cdot 10$.

Lemma 2.6 Let $1<k \leq 500, k \neq 3$ or 4 . Then any product of $k$ consecutive integers larger than 825.000 has a prime divisor $q>2 k$.

Proof. For $k=2$ the result already follows from Theorem 2.5. So we may assume that $k \geq 5$.

Let $n \in \mathbb{N}$ with $n>825000$. Assume that all prime factors in the $k$ consecutive numbers $n-k+1, \ldots, n-1, n$ are at most $2 k$.

With $\pi(x)=\#\{p$ prime $\mid p \leq x\}$, for $x \in \mathbb{R}_{+}$, we obtain

$$
\left(\frac{n}{k}\right)^{k}<\left(\begin{array}{l}
n \\
k
\end{array}\right)=\prod_{p \mid\left(\begin{array}{l}
n \\
k
\end{array}\right)} p^{b_{p}} \leq n^{\pi(2 k)}
$$

where $p^{b_{p}}$ is the maximal power of $p$ dividing $\left(\begin{array}{l}n \\ k\end{array}\right)$ (this is known to be bounded by $n$ ). Equivalently,

$$
(k-\pi(2 k)) \ln (n)<k \ln k .
$$

Note that for $5 \leq k \leq 500, k>\pi(2 k)$ (this may be checked directly). Hence, if the assumption holds then

$$
\ln (n)<\frac{k \ln (k)}{k-\pi(2 k)} .
$$

But one easily checks (e.g., with Maple) that the maximum of the function on the right hand side in the given region is bounded by 13.622 . Hence the statement of the Lemma holds for all $n>825000>e^{13.622}$. $\diamond$ 


\section{Some algorithms for hook lengths}

We write $H_{\lambda}$ for the multiset of hook lengths of a partition $\lambda$, and $F_{\lambda}$ for the set of first column hook lengths of $\lambda$, and we let $h_{\lambda}$ be the product of all the hook lengths of $\lambda$.

From now on, $\lambda$ is always a partition of $n$, of length $l$.

The following easy proposition turns out to be very useful.

Proposition 3.1 Let $p \leq n$ be a prime for which $w_{p}(\lambda)=\left\lfloor\frac{n}{p}\right\rfloor$. Let $\mu$ be the partition obtained from $\lambda$ by removing its first column. Set $A_{\lambda}=\{1, \ldots, n\} \backslash F_{\lambda}$. Let $A_{\lambda}^{p}$ and $H_{\mu}^{p}$ denote the (multi)sets of elements divisible by $p$ in the corresponding sets. Then their (multiset) cardinalities are equal:

$$
\left|A_{\lambda}^{p}\right|=\left|H_{\mu}^{p}\right|
$$

Proof. Let $F_{\lambda}^{p}$ denote the set of first column hook lengths of $\lambda$ divisible by $p$. By Lemma 2.1 we have

$$
\begin{aligned}
\left|F_{\lambda}^{p}\right|+\left|H_{\mu}^{p}\right| & =w_{p}(\lambda)=\left\lfloor\frac{n}{p}\right\rfloor \\
& =|\{j \in\{1, \ldots, n\}|p| j\}| \\
& =\left|F_{\lambda}^{p} \cup A_{\lambda}^{p}\right|=\left|F_{\lambda}^{p}\right|+\left|A_{\lambda}^{p}\right|
\end{aligned}
$$

and hence the stated equality follows. $\diamond$

Remark. Assume that $\lambda$ has maximal $p$-weight for all primes $p$. Since every "missing" first column hook length $>1$ has at least one prime factor, the sum of the number of prime factors in all hook lengths of $\mu$ should be at least the number of missing first column hook lengths. Indeed, $\left|A_{\lambda}\right|$ is the number of missing first column hook lengths and by the above proposition

$$
\left|A_{\lambda}\right| \leq \sum_{p}\left|A_{\lambda}^{p}\right|=\sum_{p}\left|H_{\mu}^{p}\right| .
$$

This is particularly useful in the cases where $\mu$ is small.

Corollary 3.2 Assume that $w_{p}(\lambda)=\left\lfloor\frac{n}{p}\right\rfloor$ for the prime $p \leq n$.

(1) Let $\mu$ be obtained from $\lambda$ by removing the first column. If $p \nmid h_{\mu}$, then $p, 2 p, \ldots,\left\lfloor\frac{n}{p}\right\rfloor p$ are first column hook lengths of $\lambda$.

(2) If $n-l(\lambda)<p$, then $p, 2 p, \ldots,\left\lfloor\frac{n}{p}\right\rfloor p$ are first column hook lengths of $\lambda$.

(3) If $n-h_{2}<p$, then $p, 2 p, \ldots,\left\lfloor\frac{n}{p}\right\rfloor p$ are first column hook lengths of $\lambda$. 
Proof. Parts (1) and (2) follow immediately from Proposition 3.1.

(3): By Corollary 2.4, all hooks of length divisible by $p$ are in the first column. With $\mu$ as in (1), $p \nmid h_{\mu}$. Hence any multiple of $p$ less than or equal to $n$ is a first column hook length, by $(1)$. $\diamond$

Assume now that $\lambda$ is partition of $n>4$, of maximal $p$-weight for all primes $p>2$.

For all $n$, we can choose primes $p_{1}, p_{2}$ such that $\frac{n}{2} \leq p_{2}<p_{1} \leq n$ [3]. By assumption, $\lambda$ must have hooks of length $p_{1}, p_{2}$, respectively.

Now by Lemma 2.3 these two hooks are either both in the first row or both in the first column of $\lambda$; w.l.o.g. we assume that they are in the first column, i.e., $p_{1}, p_{2}$ are first column hook lengths (if necessary, we replace $\lambda$ by its conjugate partition). Then, again by Lemma 2.3, any prime $q$ with $\frac{n}{2}<q \leq n$ is a first column hook length of $\lambda$.

Thus we can deduce the analogue of $[1$, Proposition 2.12] for our partition $\lambda$, i.e.:

Proposition 3.3 Suppose we have sequences of integers $s_{1}<s_{2}<\cdots<s_{r} \leq n, t_{1}<$ $t_{2}<\cdots<t_{r} \leq n$ satisfying

(i) $s_{i}<t_{i}$ for all $i$;

(ii) $s_{1}$ and $t_{1}$ are primes $>\frac{n}{2}$;

(iii) For $1 \leq i \leq r-1, s_{i+1}$ and $t_{i+1}$ contain prime factors exceeding $2 n-s_{i}-t_{i}$.

Then $s_{1}, \ldots, s_{r}, t_{1}, \ldots, t_{r}$ are first column hook lengths of $\lambda$.

For $n>3.06 \cdot 10^{8}$, we know by [1, Theorem 3.1] that we can construct sequences as in Proposition 3.3 that come close to $n$, and hence $h_{1}$ is close to $n$, namely, $n-h_{1} \leq 225$ (in fact, if $n$ is sufficiently large, we even obtain $\left.n-h_{1} \leq 2\right)$. Since 225 is too large for our purposes, we have to reduce the bound for $n-h_{1}$ further by other means. Alternatively, one could also try to prove the tight bound $n-h_{1} \leq 3$ for the improved algorithm based on the following result.

Proposition 3.4 Suppose $\lambda$ is a partition of $n \geq 5$, not a hook, which is of maximal p-weight for all primes $p>2$. Let $s_{1}<s_{2}<\cdots<s_{r} \leq n$ be a sequence of integers satisfying

(i) $s_{1}<s_{2}$ are first column hook lengths for $\lambda$;

(ii) for $3 \leq i \leq r, s_{i}$ has a prime divisor exceeding $n-s_{i-2}$.

Then $s_{1}, \ldots, s_{r}$ are first column hook lengths of $\lambda$.

Proof. We use induction on $i$ to show that $s_{i}$ is a first column hook length. Let $i \geq 3$ and assume that $s_{i-2}, s_{i-1}$ are first column hook lengths. Since $s_{i-1}>s_{i-2}$ we have $s_{i-2}=h_{j}$ for some $j>1$. Then apply Corollary 3.2 to deduce that $s_{i}$ is a first column hook length. $\diamond$ 
Note that in the algorithm derived from Proposition 3.4 we never encounter the case where the prime divisor is 2 . It is clearly a strengthening of the algorithm derived from Proposition 3.3, since it may be applied to each of the sequences $s_{i}$ and $t_{i}$. In particular, any bound for the previous algorithm is also one for this new algorithm.

Example. In the algorithms for constructing sequences according to the propositions above, we typically start with two "large" primes, which are always first column hook lengths of our special partition $\lambda$ (perhaps after conjugating $\lambda$ ). Take $n=16$. Choose $s_{1}=11, s_{2}=13$. (Note that we have hooks in $\lambda$ of this length, and - possibly after conjugation - they are both first column hook lengths.) Our previous algorithm (based on Proposition 3.3) comes to a halt here. In the new algorithm of Proposition 3.4 we can continue as $s_{3}=14$ has a prime factor $q=7>5=n-s_{1}$. Then $s_{4}=15$ has a prime factor $q=5>3=n-s_{2}$. As 16 only has the prime factor $q=2=n-s_{3}$, the algorithm terminates here. So the partitions of 16 of maximal weight for all primes $>2$ have (up to conjugation) first column hook lengths $15,14,13,11$; it is easy to check that this only holds for the partition $\left(1^{16}\right)$.

Remark 3.5 The algorithm of Proposition 3.4 is very efficient and may quickly be carried out by hand to check that $n-h_{1} \leq 3$ holds for all $n \leq 100$. It was also tested with Maple up to $n=7.5 \cdot 10^{8}$; it always gave the bound $n-h_{1} \leq 3$. Indeed, it almost always ends at $n-h_{1} \leq 1$, except for few exceptional values where it ends on the bound 2 and four cases where it ends on 3, namely for 10, 50, 100 and 15.856.204.

\section{Proofs of the main results}

We first give the proof of Theorem 1.1.

Let $\lambda=\left(\lambda_{1}, \lambda_{2}, \ldots, \lambda_{l}\right)$ be a partition of $n$, of length $l=l(\lambda)$. The following notation is fixed for $\lambda$ :

$m_{1}$ is the multiplicity of 1 as a part of $\lambda, k=\lambda_{1}-\lambda_{2}$.

$h_{1}, h_{2}, \ldots, h_{l}$ are the first column hook lengths of $\lambda, A_{\lambda}$ is the set of its "missing" first column hook lengths, $d=n-h_{1}$.

Also, as in the previous section, $\mu$ is the partition obtained from $\lambda$ by removing its first column, i.e. $\mu=\left(\lambda_{1}-1, \lambda_{2}-1, \ldots\right)$.

Let us assume

(A0) $\lambda \neq(n),\left(1^{n}\right)$.

After possibly replacing $\lambda$ by its conjugate partition (which has the same $p$-weight as $\lambda$ for all $p$ ) we are also going to assume

(A1) $l \geq \lambda_{1}$. 
Lemma 4.1 We have

(1) $k=\mu_{1}-\mu_{2}=h_{1}-h_{2}-1$.

(2) $d=|\mu|-\mu_{1}$ (the depth of $\mu$ ).

(3) $h_{1}(\mu) \leq d+k+1$.

(4) $|\mu| \leq 2 d+k$.

(5) $\lambda_{1} \leq \frac{h_{1}+1}{2} \leq l$.

(6) $h_{2} \geq m_{1}$.

(7) If $h_{2}=m_{1}$, then $\lambda$ is a hook partition. Otherwise $h_{2} \geq m_{1}+2$.

(8) $h_{2} \geq k$.

(9) If $h_{2}=k$, then $\lambda_{1}=l$ and $\lambda_{2}=1$, and hence $\lambda$ is a hook partition.

Proof. (1) and (2) are trivial. Part (3) follows from the fact that exactly $k+1$ rim nodes of the $(1,1)$-hook of $\mu$ are in the first row and at most $d$ rim nodes are outside the first row.

Since $\mu_{2} \leq d$ we get $\mu_{1} \leq d+k$ and thus (4) follows from (3).

Part (5) uses the assumption (A1) and the definition of $h_{1}$.

We have $h_{2} \geq m_{1}$ since $\lambda \neq\left(1^{n}\right)$ by (A0); thus (6) 'holds.

Part (7) is trivial.

For (8) and (9) note that $h_{2}=\lambda_{2}+l-2=\lambda_{2}+\lambda_{1}+\left(l-\lambda_{1}\right)-2$, hence $h_{2} \geq k$ is equivalent to $\left(l-\lambda_{1}\right)+2 \lambda_{2} \geq 2$. Thus the assertion follows easily from (A1) and the fact that $\lambda_{2}>0$. $\diamond$

In addition to (A0), (A1), we now make the assumption

(A2) $\lambda$ is of maximal $p$-weight for all primes $p>2$.

We consider the products

$$
\pi_{1}:=\left(h_{1}+1\right)\left(h_{1}+2\right) \cdots n, \quad \pi_{2}:=\left(h_{2}+1\right)\left(h_{1}+2\right) \cdots\left(h_{1}-1\right)
$$

having $d$ and $k$ factors, respectively (Lemma 4.1(1)); note that in the case where $\lambda$ is a hook, $d=0$, so $\pi_{1}=1$, and thus in this case we will only consider $\pi_{2}$. By definition, the factors $h_{1}+1, \ldots, n$ and $h_{2}+1, \ldots, h_{1}-1$ of $\pi_{1}, \pi_{2}$ are in $A_{\lambda}$. Thus Proposition 3.1 implies

Lemma 4.2 If $p$ is an odd prime divisor of $\pi_{1}$ or $\pi_{2}$, then $\mu$ has a hook of length $p$.

Let us now deal with the "hook case", i.e., $d=0$. Because of (A1), the leg of the hook is at least as long as its arm.

Proposition 4.3 Suppose that $\lambda=\left(k+1,1^{n-k-1}\right)$ with $1 \leq k \leq \frac{n-1}{2}$. Then $n=2^{a}+1$ for some $a \in \mathbb{N}$ and $k=1$, i.e., $\lambda=\left(2,1^{n-2}\right)$.

Proof. By Schur [7] we know that $\pi_{2}$ has a prime divisor $q>k=n-l$. If $\lambda$ has maximal $q$-weight, then by Corollary 3.2(2) $\left\lfloor\frac{n}{q}\right\rfloor q$ is a first column hook length of $\lambda$. But since $n=h_{1}>\left\lfloor\frac{n}{q}\right\rfloor q>h_{2}$, this is a contradiction. So the only critical case is when $q=2$ and 
the 2-weight is non-maximal. But when $\pi_{2}$ has only the prime divisor $q=2$, then we must have $k=1$ and $\pi_{2}=n-1$ is a 2 -power, as was to be shown. $\diamond$

Hence we may from now on assume

(A3) $\lambda$ is not a hook partition, i.e., $d>0$ and $\lambda_{2}>1$.

We have some further general relations for the parameters of $\lambda$ :

Lemma 4.4 We have

(1) $k \leq 2 d+1$.

(2) If $h_{1} \geq \frac{n}{2}$ then $d \leq 2 k+1$.

(3) $|\mu| \leq 4 d+1$.

(4) If $h_{1} \geq \frac{n}{2}$ then $|\mu| \leq 5 k+2$.

Proof. (1) is trivial if $k \leq 1$. If $k>1$, we may by Lemma 4.1(8) apply Theorem 2.5. It shows that one of the $k$ factors in $\pi_{2}$ has a prime divisor $q>\frac{3}{2} k$, or we have one of the exceptional cases. But none of these can occur:

(i) $h_{2}=2, h_{1}=5, k=2$. This is not possible, since by Lemma 4.1(5) $l \geq 3$ and then $h_{2}=2$ implies $\lambda_{2}=1$, a contradiction to (A3).

(ii) $h_{2}=7, h_{1}=10, k=2$. We get that $\left(\mu_{1}, \mu_{2}\right)$ is $(3,1)$ or $(4,2)$. Both of these partitions are 3 -cores, so we get a contradiction to Lemma 4.2.

(iii) $h_{2}=5, h_{1}=11, k=5$. Then Lemma 4.1(9) implies that $\lambda$ has to be a hook, a contradiction to (A3).

As $q$ is now a prime divisor of a number in $A_{\lambda}$, there has to be a hook in $\mu$ of length q. (Lemma 4.2.) Thus $q \leq h_{1}(\mu)$. Using Lemma 4.1(3) we obtain the inequality $\frac{3}{2} k<q \leq h_{1}(\mu) \leq d+k+1$, implying $\frac{1}{2} k<d+1$. Thus certainly $k \leq 2 d+1$. Part (2) is proved in analogy with (1) by applying Theorem 2.5 to the $d$ factors of $\pi_{1}$. Note that by assumption $d \leq h_{1}$. Then (3) and (4) follow from (1) and (2), using Lemma 4.1(4). $\diamond$

We use these relations together with results from [1] and the previous section to reduce $d=n-h_{1}$ for partitions $\lambda$ satisfying our assumptions:

Proposition 4.5 We have $d \leq 4$.

Proof. When $n \leq 5 \cdot 10^{8}$, it was already remarked before that with the new algorithm in the previous section we even get down to $d \leq 3$. (We might also use [1] where it was checked that in this range the old algorithm gets down to a bound $d \leq 4$.)

Hence we may now assume that $n>5 \cdot 10^{8}$.

Then we obtain $d \leq 225$ using [1, Theorem 3.1] (note that any bound for the old algorithm is also a bound for the new one).

We assume that $5 \leq d \leq 225$, and we want to arrive at a contradiction. By Lemma 4.4(1) we have $k \leq 451$; Lemma 4.4 also implies that the defining factors of $\pi_{1}$ and $\pi_{2}$ are greater than $10^{6}$. Indeed, these factors are bounded below by $h_{2}=n-(d+k+1) \geq$ 
$n-(225+451+1)=n-677$. We may thus apply Lemma 2.6 and get that $\pi_{1}$ has a prime divisor $q>2 d$. Thus $\mu$ must have a hook of length $q$, by Lemma 4.2. This hook has to be in the first row as there are only $d<q$ nodes in $\mu$ below the first row. By Lemma 4.1(3) we have

$$
2 d<q \leq h_{1}(\mu) \leq d+k+1
$$

and thus $d \leq k$.

We may also apply Lemma 2.6 to $\pi_{2}$ and get a prime divisor $q^{\prime}>2 k \geq 2 d$ in one of the $k$ factors in $\pi_{2}$. Hence $\mu$ must have a hook of length divisible by $q^{\prime}$ in its first row. Moreover, if $q=q^{\prime}$, then $\mu$ must have two $q$-divisible hook lengths in its first row, by Proposition 3.1. We get

$$
2 k<q^{\prime} \leq h_{1}(\mu) \leq d+1+k
$$

and thus $k \leq d$. Hence $d=k$. As both $q$ and $q^{\prime}$ are at least $2 d+1$, one of the two hook lengths is at least $2 d+2$. Thus we obtain

$$
2 d+2 \leq h_{1}(\mu) \leq d+1+k
$$

implying $d+1 \leq k$, a contradiction. $\diamond$

To finish the proof of Theorem 1.1, we now have to consider the cases where $1 \leq d \leq 4$. Here we will see the exceptional cases coming up. First we deal with the case $d=1$ :

Proposition 4.6 Suppose that $\lambda=\left(k+2,2,1^{n-k-4}\right)$ with $k \geq 0$. Then $n=4$ and $\lambda=\left(2^{2}\right), n=6$ and $\lambda=(3,2,1)$ or $n=8$ and $\lambda=\left(3,2,1^{3}\right)$.

Proof. By Lemma 4.4(1), $k \leq 3$, and by (A1), $n-k-2 \geq k+2$, hence $n \geq 2 k+4$. Here, $\mu=(k+1,1)$ and $\pi_{1}=n$.

If $k=0$, then $\pi_{2}=1$ and we use the next missing first column hook length, i.e., $\pi_{3}=n-k-3=n-3$. As $h_{\mu}=2$, both $n$ and $n-3$ have to be 2 -powers, hence $n=4$, and thus $\lambda=\left(2^{2}\right)$. If $k=1$, then $h_{\mu}=3$, and hence $\pi_{1} \pi_{2} \pi_{3}=n(n-2)(n-4)=2^{a} 3^{b}$ for some $a, b \in \mathbb{N}$. This is only possible for $n=6$ and $n=8$, and in these cases we have $\lambda=(3,2,1)$ and $\lambda=\left(3,2,1^{3}\right)$, respectively. If $k=2$, then $h_{\mu}=8$, and so $\pi_{1} \pi_{2} \pi_{3}=n(n-2)(n-3)(n-5)$ has to be a 2-power, which is impossible. If $k=3$, then $h_{\mu}=2 \cdot 3 \cdot 5$; as $\pi_{3}=(n-2)(n-3)(n-4)$ is divisible by 3 and has a prime divisor $q \geq 5$ by Theorem 2.5, then both $\pi_{1}=n$ and $\pi_{2}=n-6 \geq 4$ have to be 2-powers, which is impossible. $\diamond$

Next we deal with the case $d=2$ :

Proposition 4.7 Suppose that $\lambda=\left(k+3,3,1^{n-k-6}\right)$ or $\lambda=\left(k+2,2^{2}, 1^{n-k-6}\right)$ with $k \geq 0$. Then $n=9$ and $\lambda=\left(2^{3}, 1^{3}\right)$. 
Proof. Lemma 4.4(1) shows that $k \leq 5$. We have $\mu=(k+2,2)$ or $\mu=\left(k+1,1^{2}\right)$, respectively. We have $A_{\lambda}=\{n, n-1 ; n-3, \ldots, n-k-2$;

$n-k-4, n-k-5\}$ and $A_{\lambda}=\{n, n-1 ; n-3, \ldots, n-k-2 ; n-k-5\}$, respectively. In particular, the difference between the largest and smallest element is $k+5 \leq 10$. One can easily check that at most two elements in $A_{\lambda}$ are powers of 2 and thus we have at least $k+2$ (respectively $k+1$ ) elements of $A_{\lambda}$ divisible by odd primes. Thus $\mu$ must have at least $k+2$ (respectively $k+1$ ) hook lengths divisible by an odd prime. The former is not possible at all and the latter only for $k=0$. Here $h_{\mu}=6$ and thus $n(n-1)(n-5)=2^{a} 3^{b}$ for some $a, b \in \mathbb{N}$. This yields $n=9$, and then $\lambda=\left(2^{3}, 1^{3}\right)$. $\diamond$

Now we discuss the case $d=3$ :

Proposition 4.8 Suppose $\lambda$ is one of $\left(k+4,4,1^{n-k-8}\right),\left(k+3,3,2,1^{n-k-8}\right)$ or $(k+$ $\left.2,2^{3}, 1^{n-k-8}\right)$ with $k \geq 0$. Then $n=10$ and $\lambda=(4,3,2,1)$.

Proof. Lemma 4.4(1) shows that $k \leq 7$. We have $\mu=(k+3,3), \mu=(k+2,2,1)$ or $\mu=\left(k+1,1^{3}\right)$, respectively. Moreover, the difference between the largest missing first column hook length $n$ and the smallest one $n-k-7$ is $e=k+7 \leq 14$. At most three elements in $A_{\lambda}$ are powers of 2 , since otherwise $n=16, k=7$, a contradiction to (A1). Thus we have at least $|\mu|-3$ odd prime factors in the elements of $A_{\lambda}$. As the hook lengths in $\mu$ are at most 11, no hook length has two different odd prime divisors. Thus $\mu$ must have at least $|\mu|-3$ hook lengths divisible by an odd prime. This eliminates all possibilities for $\mu$ except $(3,2,1),\left(2,1^{3}\right)$ and $\left(1^{4}\right)$; note that in all of these cases the elements in $A_{\lambda}$ are not divisible by primes $>5$. As $\pi_{1}=n(n-1)(n-2)$ has a prime divisor $q \geq 5$ by Theorem $2.5, \mu=\left(1^{4}\right)$ cannot occur. For $\mu=\left(2,1^{3}\right)$ we have $h_{\mu}=2 \cdot 3 \cdot 5$, hence as $\pi_{1}$ is divisible by 3 and 5 , the other elements $n-4$ and $n-8$ in $A_{\lambda}$ have to be 2-powers; but this is impossible. If $\mu=(3,2,1)$, then $A_{\lambda}=\{n, n-1, n-2, n-4, n-6, n-8\}$. A parity consideration shows that this is only possible for $n=10$. $\diamond$

Proposition 4.9 The case $d=4$ is not possible.

Proof. By Remark 3.5 we may assume that $n$ is large so that we may apply Lemma 2.6 to $\pi_{2}$, if $k \neq 0,1,3,4$. We conclude as before that $\mu$ must have a hook divisible by a prime $q>2 k$ so that $2 k<q \leq h_{1}(\mu) \leq d+k+1$ yielding $k \leq d=4$. We thus have in any case $k \leq 4$ and hence $|\mu| \leq 12$ by Lemma 4.1. Applying Theorem 2.5 to $\pi_{1}$ we see that $\mu$ must have a hook of length 7 or 11. If also $k=4$ then Theorem 2.5 applied to $\pi_{2}$ shows that $\mu$ has another hook of length 7 or 11 . But these both have to be in the first row of $\mu$, and this is easily seen not to be possible. If $k=2,3$ then Theorem 2.5 shows that $\mu$ has another hook of length divisible by 5 . This is only possible for $\mu=(7,4)$, $\mu=(6,4)$ or $\mu=(5,3,1)$. If $k \leq 1$ then $h_{1}(\mu) \leq 6$, a contradiction. As $\pi_{1}$ is divisible by 3 , but $\mu=(5,3,1)$ is a 3 -core, this is excluded. For the other two possibilities, apply Theorem 2.5 to $\pi_{3}=(n-k-6)(n-k-7)(n-k-8)(n-k-9)$ to see that $\mu$ must have 
a further hook of length divisible by 7 or 11 , which is not possible. $\diamond$

Now the proof of Theorem 1.1 is complete, and with this result at hand, we may now prove Theorem 1.2 and Theorem 1.5.

Proof of Theorem 1.2. First, take a non-linear irreducible character $\chi$ of $S_{n}$; thus, $\chi=[\lambda]$, where $\lambda$ is a partition of $n$ different from $(n),\left(1^{n}\right)$. As $\left[2^{2}\right]$ is zero on transpositions, and $\left[2^{2}\right](1)=2$, we may now assume that $\lambda \neq\left(2^{2}\right)$. Hence by Theorem $1.1(1)$ there exists a prime $p$, such that $w_{p}(\lambda)<w=\left\lfloor\frac{n}{p}\right\rfloor$. By the Murnaghan-Nakayama formula (see [4, 2.4.7] or $[8,7.17 .3])$, the character then vanishes on a product of $w p$-cycles. Indeed, by Theorem 1.1(2) we may choose $p>2$ except for the partitions occurring in this case; we deal with these below to check the final assertion.

Now consider a non-linear irreducible character of $A_{n}$, labelled by $\lambda$, hence $\lambda \neq(n),\left(1^{n}\right)$, $(2,1),\left(2^{2}\right)$. If $\lambda$ is not of maximal $p$-weight for some $p>2$, then $\chi$ vanishes on the same element $\sigma$ of order $p$ as $[\lambda]$ (note that $\sigma \in A_{n}$ ). Thus, the only critical characters are the ones labelled by the exceptional partitions listed in Theorem 1.1(2), which are of non-maximal $p$-weight only for $p=2$. Of course, it suffices to consider one of a pair of conjugate partitions.

Now, if $n=2^{a}+1, a>1$, then $(n-1,1)$ is of 2 -weight $2^{a-1}-1$, and the character $[n-1,1]$ and its irreducible restriction to $A_{n}$ vanish on a product of $2^{a-1}$ transpositions (which is an element of $A_{n}$ ); note that $[n-1,1]$ is of 2 -power degree $2^{a}$. The irreducible characters of $A_{6}$ (and $S_{6}$ ) labelled by $(3,2,1)$ are zero on elements of cycle type $\left(2^{2}, 1^{2}\right)$ (these characters are of degree 8 and 16 , respectively). The character $[5,2,1]$ and its irreducible restriction to $A_{8}$ is zero on elements of cycle type $\left(2^{2}, 1^{4}\right)$ (its degree is 64$)$, and $[6,3]$ and its irreducible restriction to $A_{9}$ is zero on elements of cycle types $\left(2^{4}, 1\right)$ and $\left(3^{2}, 1^{3}\right)$. Finally, the irreducible characters of $A_{10}$ (and $\left.S_{10}\right)$ labelled by $(4,3,2,1)$ are zero on elements of cycle types $\left(2^{4}, 1^{2}\right),\left(2^{2}, 1^{6}\right)$ and $\left(3^{2}, 1^{4}\right)$. $\diamond$

Proof of Theorem 1.5. (1). The first assertion is immediate from Theorem 1.1. The second follows from the first, using that for $n \notin\{1,2,3,4,6\}$ there exist primes $p$ such that $\frac{n}{2}<p<n-1$ to see that $\left(1^{n}\right)$ is not in the principal $p$-block.

(2) This follows similarly as (1). $\diamond$

Acknowledgement. The authors would like to thank the Isaac Newton Institute for Mathematical Sciences of the University of Cambridge for its hospitality during a stay there in the frame of the programme Symmetric functions and Macdonald polynomials, where some part of the work for this article was done. We also gratefully acknowledge the support by The Danish National Research Council. 


\section{References}

[1] A. Balog, C. Bessenrodt, J. B. Olsson, K. Ono, Prime power degree representations of the symmetric and alternating groups, J. London Math. Soc. (2) 64 (2001) 344356

[2] D. Hanson, On a theorem of Sylvester and Schur, Canad. Math. Bull. 16 (1973) 195-199

[3] H. Harborth, A. Kemnitz, Calculations for Bertrand's postulate, Math. Mag. 54 (1981) 33-34

[4] G. James, A. Kerber, The representation theory of the symmetric group, Encyclopedia of Mathematics and its Applications, 16, Addison-Wesley 1981

[5] G. Malle, G. Navarro, J. B. Olsson, Zeros of characters of finite groups, J. Group Theory 3 (2000) 353-368

[6] J. B. Olsson, Combinatorics and representations of finite groups, Vorlesungen aus dem FB Mathematik der Univ. Essen, Heft 20, 1993

[7] I. Schur, Einige Sätze über Primzahlen mit Anwendungen auf Irreduzibilitätsfragen. I. Sitzungsberichte der Preuss. Akad. d. Wiss. 1929, Phys.-Math. Klasse, 125-136.

[8] R. P. Stanley, Enumerative Combinatorics, Vol. 2, Cambridge University Press, 1999 